Apr epi tant pl us gr ani set ron and dexamet hasone for prevent i on of chemot her apy-i nduced nausea and vomiting in patient $s$ with gastri c cancer treated wi th S 1 pl us ci spl at in

\begin{tabular}{|c|c|}
\hline 著者 & $\begin{array}{l}\text { Oyana Kat sunobu, Fushi da Sachi o, Kaj i } \\
\text { Masahi de, Takeda Toshi ya, K nam Shi ni chi, } \\
\text { H r ono Yasuo, Yoshi not o Kat suhi ro, Yabushi ta } \\
\text { Kazuhi sa, H r osawa H sashi, Takai Yuki, Nakano } \\
\text { Tat suo, Ki mur a H r onobu, Yasui Toshi aki, } \\
\text { Tsuneda At sushi, Tsukada Tomya, Ki noshi t a } \\
\text { Jun, Fuj i mur a Takashi, Oht a Tet suo }\end{array}$ \\
\hline $\begin{array}{l}\text { j our nal or } \\
\text { publ i cat i on titl e }\end{array}$ & Jour nal of Gast r oent er ol ogy \\
\hline vol une & 48 \\
\hline nunber & 11 \\
\hline page $r$ ange & 12341241 \\
\hline year & 2013-11- 01 \\
\hline URL & ht t p: //hdl . handl e. net /2297/33445 \\
\hline
\end{tabular}


Title:

Aprepitant plus granisetron and dexamethasone for prevention of chemotherapy-induced nausea and vomiting in patients with gastric cancer treated with S-1 plus cisplatin

Authors:

Katsunobu Oyama, Sachio Fushida, Masahide Kaji, Toshiya Takeda, Shinichi Kinami, Yasuo

Hirono, Katsuhiro Yoshimoto, Kazuhisa Yabushita, Hisashi Hirosawa, Yuki Takai, Tatsuo Nakano,

Hironobu Kimura, Toshiaki Yasui, Atsushi Tsuneda, Tomoya Tsukada, Jun Kinoshita, Takashi

Fujimura, Tetsuo Ohta, for the Digestive Disease Support Organization

Affiliation and address:

Sachio Fushida, Tomoya Tsukada, Jun Kinoshita, Takashi Fujimura, Tetsuo Ohta

Department of Gastroenterological Surgery, Kanazawa University, Kanazawa, Japan

Masahide Kaji

Department of Surgery, Toyama Prefectural Central Hospital, Toyama, Japan

Toshiya Takeda

Department of Surgery, Public Central Hospital of Matto Ishikawa, Hakusan, Japan

Shinichi Kinami

Department of General and Digestive Surgery, Kanazawa Medical University, Kahoku-gun, Japan 


\section{Yasuo Hirono}

First Department of Surgery, University of Fukui, Yoshida-gun, Japan

\section{Katsuhiro Yoshimoto}

Department of Surgery, Toyama Rosai Hospital, Uozu, Japan

\section{Kazuhisa Yabushita}

Department of Surgery, Takaoka City Hospital, Takaoka, Japan

\section{Hisashi Hirosawa}

Department of Surgery, Toyama City Hospital, Toyama, Japan

\section{Yuki Takai}

Department of Gastroenterology, Keiju Medical Center, Nanao, Japan

\section{Tatsuo Nakano}

Department of Surgery, Asanogawa General Hospital, Kanazawa, Japan

\section{Hironobu Kimura}

Department of Surgery, NTT West Kanazawa Hospital, Kanazawa, Japan

\section{Toshiaki Yasui}

Department of Surgery, Kanazawa Social Insurance Hospital, Kanazawa, Japan

\section{Atsushi Tsuneda}

Department of Surgery, Kurobe City Hospital, Kurobe, Japan

\section{Digestive Disease Support Organization (DDSO)}

35-1, Yonemaru-machi, Kanazawa, Ishikawa 921-8004, Japan 


\section{Corresponding author: Katsunobu Oyama}

Department of Gastroenterological Surgery, Kanazawa University

13-1, Takara-machi, Kanazawa, Ishikawa 920-8641, Japan

E-mail: oya-ma@staff.kanazawa-u.ac.jp

Telephone number: +81-76-265-2000

Fax number: +81-76-234-4260

Short title:

Aprepitant for CINV in GC patients 


\begin{abstract}
Background

We aimed to evaluate the efficacy of a new combination antiemetic therapy comprising aprepitant, granisetron, and dexamethasone in gastric cancer patients undergoing chemotherapy with cisplatin and
\end{abstract}

S-1.

Methods

Gastric cancer patients scheduled to receive their first course of chemotherapy with cisplatin $\left(60 \mathrm{mg} / \mathrm{m}^{2}\right)$

and S-1 (80 mg/m $\left.\mathrm{m}^{2}\right)$ were treated with a new combination antiemetic therapy aprepitant, granisetron, and dexamethasone on Day 1; aprepitant and dexamethasone on Days 2 and 3; and dexamethasone on Day 4. The patients reported vomiting, nausea, use of rescue therapy, and change in the amount of diet intake, and completed the Functional Living Index-Emesis (FLIE) questionnaire. The primary endpoint was complete response (CR; no emesis and use of no rescue antiemetics) during the overall study phase (0-120 h after cisplatin administration). The secondary endpoints included complete protection (CP; CR plus no significant nausea); change in the amount of diet intake; and the impact of chemotherapy-induced nausea and vomiting (CINV) on daily life during the overall, acute (0-24 h), and delayed (24-120 h) phases.

Results

Fifty-three patients were included. CR was achieved in $88.7 \%, 98.1 \%$, and $88.7 \%$ of patients in the overall, acute, and delayed phases, respectively. The corresponding rates of CP were $67.9 \%, 96.2 \%$, and 67.9\%. Approximately half of the patients had some degree of anorexia. FLIE results indicated that 
79.5\% of patients reported "minimal or no impact of CINV on daily life".

Conclusions

Addition of aprepitant to standard antiemetic therapy was effective in gastric cancer patients undergoing

treatment with cisplatin and S-1.

Keywords: aprepitant, gastric cancer, CINV, anorexia, QOL 


\section{Introduction}

Chemotherapy-induced nausea and vomiting (CINV) is a common adverse event observed in more than $90 \%$ of patients treated with highly emetogenic antitumor agents [1]. CINV is one of the greatest fears of patients beginning chemotherapy [2, 3]. Inadequate control of CINV can lead to dehydration, malnutrition, and electrolyte imbalance. These symptoms impair functional activity and quality of life (QOL) of patients, increase the use of healthcare resources, and may occasionally cause treatment delay or discontinuation [4-6].

Generally, CINV persists approximately for 5 days after administration of emetogenic antitumor agents. CINV occurring within the first $24 \mathrm{~h}$ has been defined as acute and that occurring after more than $24 \mathrm{~h}$ as delayed [7]. The incidence and severity of CINV are affected by a number of factors, including the chemotherapeutic regimen-related factors such as the agent, dose, and schedule and patient-related factors such as age, sex, and history of alcohol use [8]. Of all the known predictive factors for CINV, the dose and intrinsic emetogenicity of a given chemotherapeutic agent are the important factors [9, 10]. Cisplatin, one of the strongest emetogenic antitumor agents, can cause both acute and delayed emesis. The incidence of CINV induced by cisplatin is $98 \%$ in the acute phase and $77 \%$ in the delayed phase after administration of $\geq 50 \mathrm{mg} / \mathrm{m}^{2}$ of cisplatin without preventive treatment [11].

Corticosteroids have been used as effective antiemetic agents for CINV for a long time [12]. They are effective for both acute and delayed emesis. The introduction of selective serotonin (5-hydroxytriptamine-3, [5- $\left.\mathrm{HT}_{3}\right]$ ) receptor antagonists (RA) revolutionized the control of CINV. 5-HT ${ }_{3}$ RAs are used for moderate to highly emetogenic chemotherapy, and these agents exert protective 
effects mainly in acute emesis. Although combination of corticosteroids and 5- $\mathrm{HT}_{3} \mathrm{RA}$ has been used as standard therapy for management for CINV, more than $50 \%$ of patients continue to vomit in response to highly emetogenic chemotherapy such as high-dose cisplatin [13]. This combination therapy prevents vomiting in the acute phase, but appears to lack efficacy in the delayed phase [14-16].

The neurokinin-1 ( $\left.\mathrm{NK}_{1}\right) \mathrm{RA}$ represents the newest class of antiemetic agents for the prevention of CINV. $\mathrm{NK}_{1}$ receptors regulate the vomiting reflex, which is predominant during delayed phase [17]. Aprepitant, a new selective $\mathrm{NK}_{1} \mathrm{RA}$, was the first available agent in this class, dramatically prevented CINV. Prospective phase III trials performed using highly emetogenic chemotherapy led to the approval of aprepitant [18-20]. In each trial, addition of aprepitant to the standard antiemetic therapy, 5- $\mathrm{HT}_{3} \mathrm{RA}$ and corticosteroid, controlled emesis by further 15 to $20 \%$.

The availability of new antiemetic agents has contributed to substantial improvements in control of emesis. A single agent cannot provide complete protection against various phases of emesis, while a combination of antiemetic agents such as $\mathrm{NK}_{1} \mathrm{RA}$ and 5- $\mathrm{HT}_{3} \mathrm{RAs}$ and corticosteroids can result in better prevention of CINV. Recently, several groups such as the Multinational Association of Supportive Care in Cancer (MASCC)/the European Society for Medical Oncology (ESMO), the American Society of Clinical Oncology (ASCO), and the National Comprehensive Cancer Network (NCCN) have published and updated international antiemetic guidelines [8, 21, 22]. In 2010, Japanese Society of Clinical Oncology (JSCO) also published the antiemetic guideline [23]. They recommend the use of $\mathrm{NK}_{1} \mathrm{RAs}$ in combination with $5-\mathrm{HT}_{3} \mathrm{RAs}$ and corticosteroid to prevent CINV induced by a highly and moderately emetogenic chemotherapy. 
Gastric cancer is one of the major causes of cancer death worldwide, and chemotherapy is the main treatment option for patients with advanced gastric cancer. To date, cisplatin plus fluoropyrimidine is a standard chemotherapeutic regimen for advanced gastric cancer, which definitely induces CINV. In the SPIRITS trial, a large phase III trial of cisplatin plus S-1 (an orally administrated 5-fluorouracil analog) for advanced gastric cancer, emesis occurred in 36\% of patients and nausea in $67 \%$ of patients [24].

No studies have been performed with the new standard antiemetic regimen with a focus on gastric cancer patients treated with cisplatin-based chemotherapy. Some issues about the approval of aprepitant for patients with gastric cancer remain to be addressed. First, no study on CINV has been performed with a focus on gastric cancer. Furthermore, although the standard dose of cisplatin in S-1 plus cisplatin chemotherapy for gastric cancer is $60 \mathrm{mg} / \mathrm{m} 2$, the dose administered to gastric cancer patients in previous studies was $\geq 70 \mathrm{mg} / \mathrm{m} 2$ [18-20]. Therefore, the need for aprepitant is not known. Therefore, we performed a prospective observational study to evaluate the efficacy of a combination antiemetic therapy with aprepitant, granisetron, and dexamethasone in Japanese gastric cancer patients undergoing an initial chemotherapy cycle with cisplatin plus S-1. In addition, to our knowledge, no previous studies have reported the incidences of CINV in the initial cycle of chemotherapy, the incidence and degree of anorexia, and the impact of CINV on QOL with a focus on gastric cancer. Our results may be point of reference for CINV in gastric cancer patients. 


\section{Methods}

\section{Design}

This study was a multi-institutional, prospective, observational, non-comparative study involving 17 institutions of the Digestive Disease Support Organization (DDSO). We performed an observational study because a 3-drug combination therapy involving aprepitant is the recommended antiemetic prophylaxis in patients receiving cisplatin-based chemotherapy. Patients gave written informed consent. The protocol was approved by the institutional review board at each participating center, and the study was performed in accordance with the principles of the Declaration of Helsinki. (Clinical trial ID: UMIN000004175)

\section{Eligibility criteria}

High or moderate emetogenic chemotherapy-naive patients who were scheduled to receive their first course of chemotherapy with cisplatin $\left(60 \mathrm{mg} / \mathrm{m}^{2}\right)$ and S-1 $\left(80 \mathrm{mg} / \mathrm{m}^{2}\right)$ for pathologically confirmed gastric cancer were eligible. Patients were required to be $\geq 20$ years of age and to have an Eastern Cooperative Oncology Group (ECOG) Performance Status of 0 to 2. Patients with any vomiting, retching, or nausea (National Cancer Institute $[\mathrm{NCI}] \geq$ grade I) $24 \mathrm{~h}$ before treatment or those using any drug with potential antiemetic efficacy in the $48 \mathrm{~h}$ before chemotherapy were ineligible. In addition, exclusion criteria included the following: radiation therapy to the abdomen or pelvis any time 1 week before treatment, a symptomatic primary or metastatic central nervous system (CNS) malignancy, a risk of vomiting for other reasons (epilepsy, active peptic ulcer, and gastrointestinal obstruction), and any uncontrolled disease other than malignancy that may pose an unwarranted risk as determined by the 
investigator.

\section{Chemotherapy}

All patients received S-1 plus cisplatin therapy according to SPIRITS trial [24]; S-1 plus cisplatin is the standard chemotherapeutic regimen for advanced gastric cancer in Japan. S-1 (80 mg/m²) was administered orally twice daily for the first 3 weeks of a 5-week cycle. Cisplatin was administered as an intravenous infusion of $60 \mathrm{mg} / \mathrm{m}^{2}$ on day 8 of each cycle.

\section{Antiemetic treatment}

All patients received the following antiemetics: oral aprepitant $125 \mathrm{mg} 60 \mathrm{~min}$ before cisplatin infusion plus intravenous dexamethasone $9.9 \mathrm{mg}$ and intravenous granisetron $3 \mathrm{mg} 30 \mathrm{~min}$ before cisplatin infusion on Day 1, oral aprepitant $80 \mathrm{mg}$ once daily each morning and oral dexamethasone $8 \mathrm{mg}$ bid on Days 2 and 3, and oral dexamethasone $8 \mathrm{mg}$ bid on Day 4. This combination of antiemetics is recommended in JSCO Guidelines for Antiemetics in Oncology 2010 [23]. Patients were given a prescription for a rescue antiemetic to be used only when nausea and vomiting developed during the 120-h observation period.

\section{Response definitions}

The observation period was divided in 3 distinct phases: acute, 0 to $24 \mathrm{~h}$; delayed, 24 to $120 \mathrm{~h}$; and overall, 0 to $120 \mathrm{~h}$ after injection of cisplatin. During the 120-h assessment period after the initiation of cisplatin infusion, patients were required to maintain a diary and record the number and timing of any episodes of vomiting or retching; the frequency and timing of use of rescue antiemetics; and the degree of nausea using a 4-point categorical scale ( 0 , none; 1 , mild; 2 , moderate; 3 , severe). Volume changes of diet 
intake were recorded by patients every day on Days 1 to 5 as \% volume of diet after treatment compared to that before the initiation of chemotherapy as baseline. Patients responded to the Functional Living Index-Emesis (FLIE) questionnaire once a day from Days 1 to 5, which captured information about the effect of CINV on the daily lives of the patients.

The primary endpoint was the proportion of patients achieving CR (defined as no emesis and no rescue antiemetics use) during the overall study phase. No vomiting was defined as no vomiting, retching, or dry heaves. Secondary endpoints included the rate of CP (meet criteria for CR plus no significant nausea [nausea score, 0 and 1 , nausea that does not interfere with the normal activities of the patient]); volume change of diet intake; no vomiting; no nausea; the impact of CINV on daily life (as measured by a FLIE) during the overall, acute, and delayed phases.

The FLIE instrument is a patient-completed multidimensional questionnaire to evaluate the QOL [25]. Japanese version of the FLIE was used in this study, which was reported useful in assessing the impact of CINV on the QOL of Japanese patients [26]. The FLIE questionnaire contains a validated 18-item visual analogue scale (VAS)-based, patient-reported outcome measure that captures information about the effect of CINV on the daily lives of the patients. FLIE has separate domains for the impact of nausea and vomiting on the daily function of patients. Each item is answered using a 1- to 7-point VAS. Each item scales from 7 to 1 (“not at all” to "a great deal”). Average score $>6$ points; total score $>108$ out of a maximum possible 126 points; each domain score $>54$, defined as "minimal or no impact of CINV on daily life.” Safety was evaluated on the basis of physical examination, including vital signs, routine clinical 
laboratory tests, and adverse event reporting. Toxicity grades were assessed using the NCI Common Terminology Criteria for Adverse Events (NCI-CTCAE) v4.0.

(http://ctep.cancer.gov/forms/CTCAEv4.pdf). The adverse events were determined by the investigator to be possibly, probably, or definitively related to the study drug.

\section{Statistical analysis}

The incidence of CINV in the target population of this study is not clear; therefore, no definitive reference is available to calculate the standard deviation and sample size. In previous studies using aprepitant, the rates of CR were improved by approximately $20 \%$ after the addition of aprepitant to standard therapy. The SPIRITS trial showed that emesis was observed in $36 \%$ of patients, and nausea was observed in $67 \%$ (Grade 3/4, 11\%) of patients. The incidence of emesis and nausea requiring treatment was assumed about 50\%, which accounted for 36\% (emesis) plus 11\% (Grade 3/4 nausea). To set an expected CR rate of $70 \%$ and a threshold CR rate of $60 \%$, a sample size of 50 subjects was estimated to be required to provide a power of $80 \%$ assuming a two-sided test and an overall significance level of 0.05 . Assuming that approximately $10 \%$ of subjects would be withdrawn or drop out, we selected a target sample size of 55 subjects. 


\section{Results}

\section{Patient characteristics}

Between January 2011 and May 2012, 56 patients were enrolled at 13 centers in Japan. Of the 56 patients, 53 satisfied the eligibility criteria; the 3 patients who were excluded did not receive cisplatin injection, and they were included in the efficacy analyses. The basic characteristics of the patients are described in Table 1. Most patients were men (90.6\%), and the median age of the patients was 65 years.

\section{Antiemetic outcome}

Antiemetic outcome is shown in Figure 1 and Table 2. The primary endpoint of CR for the overall study phase was achieved in 47 (88.7\%) patients. Acute and delayed CR rates were 98.1\% (52/53) and 88.7\% (47/53), respectively. These results were considerably superior to the expected CR rate of 70\%. CP rates for the overall, acute, and delayed study phases were 67.9\% (36/53), 96.2\% (51/53), and 67.9\% (36/53), respectively. "No vomiting” rates for the overall, acute, and delayed study phases were 92.5\% (49/53), 98.1\% (52/53), and 92.5\% (49/53), and “No nausea” rates for the overall, acute, and delayed study phases were 64.1\% (34/53), 92.4\% (49/53), and 66.0\% (35/53), respectively. Overall, 19 (35.9\%) patients experienced some degree of nausea; mild nausea in 11 (20.8\%) patients, moderate nausea in 7 (13.2\%) patients, and severe nausea in 1 (1.9\%) patient.

\section{Assessment of the QOL (Table 3)}

Of the 53 eligible patients, 49, 50, and 49 patients answered the FLIE questionnaire in the overall, acute, and delayed phases, respectively. In the overall period of 5 days, 39 patients (79.5\%) had a total FLIE score of more than 108, and 37 (75.5\%) and 44 (89.8\%) patients, respectively, had a nausea domain 
score and vomiting domain score of more than 54, and reported "minimal or no impact of CINV on daily life.” Further, in the acute phase, 49 patients (98.0\%) had a total FLIE score of more than 108, and 48 (96.0\%) and 50 (100\%) patients, respectively, had a nausea domain score and vomiting domain score of more than 54, and reported "minimal or no impact of CINV on daily life.” Finally, in the delayed phase, 39 patients (79.5\%) had a total FLIE score of more than 108, and 37 (75.5\%) and 44 (89.8\%) patients, respectively, had a nausea domain score and vomiting domain score of more than 54, and reported “minimal or no impact of CINV on daily life.

\section{Diet intake (Figure 2)}

Approximately half of the patients had some degree of anorexia, and the decrease in oral intake was predominant in the delayed phase. Volume of diet intake was reduced to half in $30 \%$ of patients; in addition, $10 \%$ of the patients could not consume any food and beverage in the delayed phase.

\section{Safety}

Overall, antiemetic therapy was well-tolerated. Adverse events considered by the investigator to be possibly, probably, or definitely related to the study drug were anorexia in 7 (13.2\%) patients, diarrhea in 4 (7.5\%) patients; hiccups in 3 (5.7\%) patients, and constipation in 1 (1.9\%) patient. No serious adverse events appeared to be related to the study drug. 


\section{Discussion}

We have reported the results of prospective phase III trials performed using highly emetogenic chemotherapy that led to the approval of aprepitant [18-20]. Hesketh PJ and colleagues found that compared to the standard regimen, addition of aprepitant to the standard regimen improved the CR rates in overall (52\% versus $72 \%$ ), acute (78\% versus $89 \%$ ), and delayed phases (55\% versus 75\%) [18]; similarly, Poli-Bigelli and colleagues reported enhanced CR rates, in overall (43\% versus 62\%), acute (68\% versus 82\%), and delayed phases (46\% versus 67\%), respectively [19]. Schmoll HJ and co-workers showed that the CR rates were higher after addition of aprepitant in the overall (72\% versus $61 \%$ ), acute (88\% versus $79 \%$ ), and delayed phases (74\% versus 63\%) [20]. These results indicate that addition of aprepitant had an obvious therapeutic advantage.

Our study included a cohort of patients with solid cancer, who were scheduled to receive the chemotherapy containing cisplatin $\geq 70 \mathrm{mg} / \mathrm{m}^{2}$. The standard chemotherapy for advanced gastric cancer in Japan is $60 \mathrm{mg} / \mathrm{m}^{2}$ cisplatin; however, to our knowledge, no study has reported the efficacy of cisplatin plus S-1 therapy in a gastric cancer patient. In the SPIRITS trial, a large phase III trial of cisplatin plus S-1 for advanced gastric cancer, emesis occurred in 36\% of patients and nausea in $67 \%$ of patients [24]. A large population of patients has CINV. Therefore, we performed this observational study to evaluate the efficacy of a new combination antiemetic therapy involving addition of aprepitant to the standard antiemetic therapy, focusing on Japanese patients with gastric cancer who received an initial cycle of cisplatin plus S-1.

In the present study, a combination of aprepitant and granisetron and dexamethasone (recommended 
regimen according to JSCO Guidelines for Antiemetics in Oncology 2010 [23]) showed that CR ratios were $88.7 \%, 98.1 \%$, and $88.7 \%$ in patients in the overall, acute, and delayed phases, respectively. Approximately $90 \%$ of patients with gastric cancer receiving an initial cycle of cisplatin plus S-1 chemotherapy were free from emesis. This result is similar to that of other aprepitant-containing antiemetic studies. The imbalance of male-to-female ratio was occurred in this study unexpectedly, the sample size of the study may affect the imbalance of gender. Although the patients in our study were biased towards male gender and elderly patients resistant to CINV, the results our study were excellent. CP rates were $67.9 \%, 96.2 \%$, and $67.9 \%$ in patients in the overall, acute, and delayed phases, respectively. Control of nausea was not achieved in approximately 30\% patients in the delayed phase; therefore, nausea was not as well-controlled as vomiting. These results indicate that this triple-drug combination therapy is not effective in controlling nausea in the delayed phase. Generally, clinicians underestimate the incidence of nausea, which is not as well-controlled as vomiting. Clinicians should pay more attention to nausea.

We use the FLIE questionnaire to assess the patient-reported impact of CINV on the QOL [25]. In this study, results of the FLIE questionnaire showed that a high percentage of patients reported "minimal or no impact of CINV on daily life.” A reduction in the QOL was observed in approximately $20 \%$ more patients; the score in the nausea domain was inferior to the score in the vomiting domain, and the score in delayed phase was inferior to the score in the acute phase. The principal reason for reduction in the QOL was nausea, particularly in the delayed phase. Thus, new strategies for better control of nausea are required. 
Moreover, approximately half of the patients showed a reduction in dietary intake, particularly in the delayed phase. Generally, loss of oral intake was considered as a symptom interrelated with the degree of nausea. In this study, the rate of anorexia was higher than the incidence of nausea. Loss of oral intake is thought to be the result of several distresses such as nausea, appetite loss, and decline in motivation to eat correlated with chemotherapy. Malnutrition in cancer patients affects the overall condition of the patient; it increases the number of complications and adverse effects of chemotherapy and reduces the QOL. Therefore, control of anorexia is an important consideration.

Aprepitant used in combination with standard antiemetic therapy (5- $\mathrm{HT}_{3} \mathrm{RA}$ and corticosteroid) was well-tolerated and very effective in preventing CINV; therefore, it should be considered as a new standard of antiemetic prophylaxis for patients with gastric cancer treated with cisplatin plus S-1 chemotherapy. Although aprepitant is an important in controlling CINV, uncontrolled vomiting and inadequately controlled nausea continue to be the major problems in some patients. It is noteworthy that $30 \%$ of patients had uncontrolled nausea and half of the patients had anorexia in our study. The $\mathrm{NK}_{1} \mathrm{RA}$ may have less impact on the nausea component of CINV. Further improvement in the prevention of CINV will require more effective anti-nausea treatments. The control of nausea does not take precedence over the control of vomiting because the physiology of nausea is not well-understood [27] and the difficulty in measuring this subjective symptom; patients confuse nausea with anorexia, fatigue, or pyrosis [3]. Palonosetron, the second-generation 5- $\mathrm{HT}_{3} \mathrm{RA}$ differs from the older 5- $\mathrm{HT}_{3} \mathrm{RAs}$ in its prolonged half-life (approximately $40 \mathrm{~h}$ ) and its substantially greater binding affinity for the $5-\mathrm{HT}_{3}$ receptor [28]. It is a 
potent $5-\mathrm{HT}_{3} \mathrm{RA}$ that is more favorable compared to the first-generation $5-\mathrm{HT}_{3} \mathrm{RAs}$ in terms of effectiveness and safety. Moreover, palonosetron specifically improves nausea control [29]. Thus, the question that whether palonosetron should be the preferred $5-\mathrm{HT}_{3} \mathrm{RA}$ when aprepitant is used should be addressed. To date, limited information is available about the combination of palonosetron and aprepitant [30-32]. The newest combination antiemetic therapy with palonosetron and aprepitant plus corticosteroid may lead to further improvement in the control of CINV. We performed this study for confirming the effect of the newest combination antiemetic therapy on CINV in gastric cancer patients receiving cisplatin plus S-1 chemotherapy.

Despite limited sample size, our study has several important results. New combination antiemetic therapy involving addition of aprepitant to the standard antiemetic therapy was effective in Japanese patients with gastric cancer who received an initial cycle of cisplatin plus S-1. CINV was controlled as indicated by the result that a majority of the patients maintained their QOL. Our results indicate that this antiemetic regimen should be a recommended therapy for this population. Despite this effective antiemetic prophylaxis, there was room for improvement in controlling nausea and anorexia; therefore, further therapeutic intervention is required. In addition, our results indicate the incidences of CINV, particularly in patients receiving an initial cycle of chemotherapy; the incidence and degree of anorexia; the impact of CINV on the QOL of gastric cancer patients receiving chemotherapy. Our result serves as a useful benchmark for future studies on CINV.

\section{Conflict of interest}


The authors declare that they have no conflict of interest.

\section{References}

1. Bender CM, McDaniel RW, Murphy-Ende K,Pickett M, Rittenberg CN, Rogers MP,et al. Chemotherapy-inducednausea and vomiting. Clin J Oncol Nurs. 2002; 6: 94-102.

2. de Boer-Dennert M, de Wit R, Schmitz PI, Djontono J, v Beurden V, Stoter G, et al. Patient perceptions of the sideeffects of chemotherapy: the influence of 5HT3 antagonists. Br J Cancer. 1997;76:1055-61.

3. Hickok JT, Roscoe JA, Morrow GR, King DK, Atkins JN, Fitch TR. Nausea and emesis remain significant problems of chemotherapy despite prophylaxis with 5-hydroxytryptamine-3 antiemetics: a University of Rochester James P. Wilmot Cancer Center Community Clinical Oncology Program Study of 360 cancer patients treated in the community. Cancer. 2003;97:2880-6.

4. Lindley CM, Hirsch JD, O’Neill CV, Transau MC, Gilbert CS, Osterhaus JT. Quality of life consequences of chemotherapy-induced emesis. Qual Life Res. 1992;1:331-40.

5. Bloechl-Daum B, Deuson RR, Mavros P, Hansen M, Herrstedt J. Delayed nausea and vomiting continue to reduce patients' quality of life after highly and moderately emetogenic chemotherapy despite antiemetic treatment. J Clin Oncol. 2006;24: 4472-8.

6. Ihbe-Heffinger A, Ehlken B, Bernard R, Berger K, Peschel C, Eichler HG, et al. The impact of delayed chemotherapy-induced nausea and vomiting on patients, health resource utilization and costs in German cancer centers. Ann Oncol. 2004;15:526-36.

7. Tavorath R, Hesketh PJ. Drug treatment of chemotherapy-induced delayed emesis. 
Drugs.1996;52:639-48.

8. Ettinger DS, Bierman PJ, Bradbury B, Comish CC, Ellis G, Ignoffo RJ, et al. Antiemesis. J Natl Compr Canc Netw. 2007;5:12-33.

9. du Bois A, Meerpohl HG, Vach W, Kommoss FG, Fenzl E, Pfleiderer A. Course, patterns, and risk-factors for chemotherapy-induced emesis in cisplatin-pretreated patients: a study with ondansetron. Eur J Cancer. 1992;28:450-7.

10. Hesketh PJ. Defining the emetogenicity of cancer chemotherapy regimens: relevance to clinical practice. Oncologist. 1999;4:191-6.

11. Kris MG, Cubeddu LX, Gralla RJ,Cupissol D, Tyson LB, Venkatraman E, et al. Are more antiemetic trials with a placebo necessary? Report of patient data from randomized trials of placebo antiemetics with cisplatin. Cancer. 1996; 78: 2193-8.

12. Aapro MS, Alberts DS. High-dose dexamethasone for prevention of cisplatin-induced vomiting. Cancer Chemother Pharmacol. 1981;7:11-4.

13. Gralla RJ, Osoba D, Kris MG,Kirkbride P, Hesketh PJ, Chinnery LW, et al. Recommendations for the use of antiemetics: evidence-based, clinical practice guidelines. J Clin Oncol. 1999; 17: 297194.

14. Jantunen IT, Kataja VV, Muhonen TT. An overview of randomised studies comparing 5-HT3 receptor antagonists to conventional anti-emetics in the prophylaxis of acute chemotherapy-induced vomiting. Eur J Cancer. 1997; 33: 66-74.

15. Latreille J, Pater J, Johnston D, Laberge F, Stewart D, Rusthoven J, et al. Use of dexamethasone 
and granisetron in the control of delayed emesis for patients who receive highly emetogenic chemotherapy. National Cancer Institute of Canada Clinical Trials Group. J Clin Oncol. 1998; 16: $1174-8$.

16. Tsukada H, Hirose T, Yokoyama A,Kurita Y. Randomised comparison of ondansetron plus dexamethasone with dexamethasone alone for the control of delayed cisplatin-induced emesis. Eur J Cancer. 2001; 37: 2398-404.

17. Hesketh PJ, Van Belle S, Aapro M,Tattersall FD, Naylor RJ, Hargreaves R, et al. Differential involvement of neurotransmitters through the time course of cisplatin-induced emesis as revealed by therapy with specific receptor antagonists. Eur J Cancer. 2003; 39: 1074-80.

18. Hesketh PJ, Grunberg SM, Gralla RJ, Warr DG, Roila F, de Wit R, et al. The oral neurokinin-1 antagonist aprepitant for the prevention of chemotherapy-induced nausea and vomiting: a multinational, randomized, double-blind, placebo-controlled trial in patients receiving high-dose cisplatin — the Aprepitant Protocol 052 Study Group. J Clin Oncol. 2003;21:4112-9.

19. Poli-Bigelli S, Rodrigues-Pereira J, Carides AD, Julie Ma G, Eldridge K, Hipple A,et al.; Aprepitant Protocol 054 Study Group. Addition of the neurokinin-1 receptor antagonist aprepitant to standard antiemetic therapy improves control of chemotherapy induced nausea and vomiting: results from a randomized, double-blind, placebo-controlled trial in Latin America. Cancer. 2003;97:3090-8.

20. Schmoll HJ, Aapro MS, Poli-Bigelli S, Kim HK, Park K, Jordan K, et al. Comparison of an aprepitant regimen with a multiple-day ondansetron regimen, both with dexamethasone, for 
antiemetic efficacy in high-dose cisplatin treatment. Ann Oncol. 2006;17:1000-6.

21. Roila F, Herrstedt J, Aapro M, Gralla RJ, Einhorn LH, Ballatori E, et al. Guideline update for MASCC and ESMO in the prevention of chemotherapy and radiotherapy-induced nausea and vomiting: results of the Perugia consensus conference. Ann Oncol. 2010; 21 (Supplement 5): v232-43.

22. Ethan Basch, Ann Alexis Prestrud, Paul J. Hesketh, Mark G. Kris, Petra C. Feyer, Mark R. Somerfield, et al. Antiemetics: American Society of Clinical Oncology Clinical Practice Guideline Update. J Clin Oncol. 2011;29: 4189-98.

23. JSCO Guidelines for Antiemetics in Oncology 2010. $1^{\text {st }}$ ed. Tokyo: Kanahara shuppann; 2010.

24. Koizumi W, Narahara H, Hara T, Takagane A, Akiya T, Takagi M, et al. S-1 plus cisplatin versus S-1 alone for first-line treatment of advanced gastric cancer (SPIRITS trial): a phase III trial. Lancet Oncol. 2008;9:215-21.

25. Martin AR, Pearson JD, Cai B, Elmer M, Horgan K, Lindley C. Assessing the impact of chemotherapy-induced nausea and vomiting on patients' daily lives: a modified version of the Functional Living Index-Emesis (FLIE) with 5-day recall. Support Care Cancer. 2003;11: 522-7.

26. Satou A, Yamazaki T, Nukariya N, Nakamachi M, Shimada K, Matsukawa M, et al. Development of a Japanese version of the FLIE. Gan To Kagaku Ryoho. 2002; 29: 281-91.

27. Stephenson J, Davies A. An assessment of aetiology-based guidelines for the management of nausea and vomiting in patients with advanced cancer. Support Care Cancer. 2006;14: 348-53. 
28. Tonini G, Vincenzi B, Santini D. New drugs for chemotherapy-induced nausea and vomiting: focus on palonosetron. Expert Opin Drug Metab Toxicol. 2005;1: 143-9.

29. Gralla R, Lichinitser M, Van Der Vegt S, Sleeboom H, Mezger J, Peschel C, et al. Palonosetron improves prevention of chemotherapy-induced nausea and vomiting following moderately emetogenic chemotherapy: results of a double-blind randomized phase III trial comparing single doses of palonosetron with ondansetron. Ann Oncol. 2003; 14:1570-7.

30. Grote T, Hajdenberg J, Cartmell A Ferguson S, Ginkel A, Charu V.Combination therapy for chemotherapy-induced nausea and vomiting in patients receiving moderately emetogenic chemotherapy: palonosetron, dexamethasone and aprepitant. J Support Oncol.2006; 4:403-8.

31. Herrington JD, Jaskiewicz AD, Song J. Randomized, placebo-controlled, pilot study evaluating aprepitant single dose plus palonosetron and dexamethasone for the prevention of acute and delayed chemotherapy-induced nausea and vomiting. Cancer. 2008; 112:2080-7.

32. Grunberg SM, Dugan M, Muss H Wood M, Burdette-Radoux S, Weisberg T, Siebel M,et al. Effectiveness of a single-day three-drug regimen of dexamethasone, palonosetron, and aprepitant for the prevention of acute and delayed nausea and vomiting caused by moderately emetogenic chemotherapy. Support Care Cancer. 2009; 17:589-94. 


\section{Figure legends}

Figure 1 - Percentage of patients with complete response (CR) and complete protection (CP).

CR for the overall, acute, and delayed phases was achieved in $88.7 \%, 98.1 \%$, and $88.7 \%$ of patients. CP rates for the overall, acute, and delayed phases were $67.9 \%, 96.2 \%$, and $67.9 \%$, respectively.

Figure 2 - Decrease in diet intake compared to that before initiation of chemotherapy.

Approximately half of the patients had some degree of anorexia; the decrease in oral intake was predominant in the delayed phase. 
Table 1. Patient characteristics

\begin{tabular}{lcc}
\multicolumn{2}{c}{ Characteristics } & Number of patients \\
\hline All & & 53 \\
\hline Age (range, 50 to 81 years; median, 65 & $-65: 66-$ & $29: 24$ \\
years) & Male:Female & $48: 5$ \\
Gender & $0: 1$ & $36: 17$ \\
Performance status & II:III: IV:recurrence & $2: 13: 23: 14$ \\
Clinical stage of gastric cancer (TNM) & None:several times:almost daily & $25: 5: 23$ \\
Alcoholic drinks & negative:positive & $14: 39$ \\
History of chemotherapy & negative:positive & $53: 0$ \\
History of chemotherapy-induced & & \\
nausea and vomiting (CINV) & negative:positive & $52: 1$ \\
History of morning sickness & negative:positive & $52: 1$ \\
History of motion sickness &
\end{tabular}

Table 2. Percentage of patients who achieved efficacy endpoint

\begin{tabular}{lccc} 
& Overall $(0-120 \mathrm{~h})$ & Acute $(0-24 \mathrm{~h})$ & Delayed $(24-120 \mathrm{~h})$ \\
\hline Complete response & $88.7 \%$ & $98.1 \%$ & $88.7 \%$ \\
Complete protection & $67.9 \%$ & $96.2 \%$ & $67.9 \%$ \\
No use of rescue therapy & $96.2 \%$ & $100 \%$ & $96.2 \%$ \\
No vomiting & $92.5 \%$ & $98.1 \%$ & $92.5 \%$ \\
No nausea & $64.1 \%$ & $92.4 \%$ & $66.0 \%$ \\
No significant nausea & $69.8 \%$ & $98.1 \%$ & $69.8 \%$ \\
\hline
\end{tabular}

Table 3. Quality of life (QOL) assessment based on functional living index-emesis (FLIE) questionnaire

\begin{tabular}{|c|c|c|c|c|c|c|c|c|c|}
\hline \multirow[b]{3}{*}{ FLIE item } & \multicolumn{3}{|c|}{ Overall (0-120 h) } & \multicolumn{3}{|c|}{ Acute $(0-24 \mathrm{~h})$} & \multicolumn{3}{|c|}{ Delayed (24-120 h) } \\
\hline & \multicolumn{2}{|c|}{ Number of patients } & \multirow[b]{2}{*}{$\%$} & \multicolumn{2}{|c|}{ Number of patients } & \multirow[b]{2}{*}{$\%$} & \multicolumn{2}{|c|}{ Number of patients } & \multirow[b]{2}{*}{$\%$} \\
\hline & Total & NIDL & & Total & NIDL & & Total & NIDL & \\
\hline FLIE total score & 49 & 39 & 79.5 & 50 & 49 & 98.0 & 49 & 39 & 79.5 \\
\hline nausea domain total score & 49 & 37 & 75.5 & 50 & 48 & 96.0 & 49 & 37 & 75.5 \\
\hline vomiting domain total score & 49 & 44 & 89.8 & 50 & 50 & 100 & 49 & 44 & 89.8 \\
\hline
\end{tabular}

NIDL, No or minimal impact on daily life. Defined as domain total FLIE score of more than 54 or total FLIE score more than 108 
Figure 1. Percentage of patients with complete response and complete protection

$\%$ of Patients
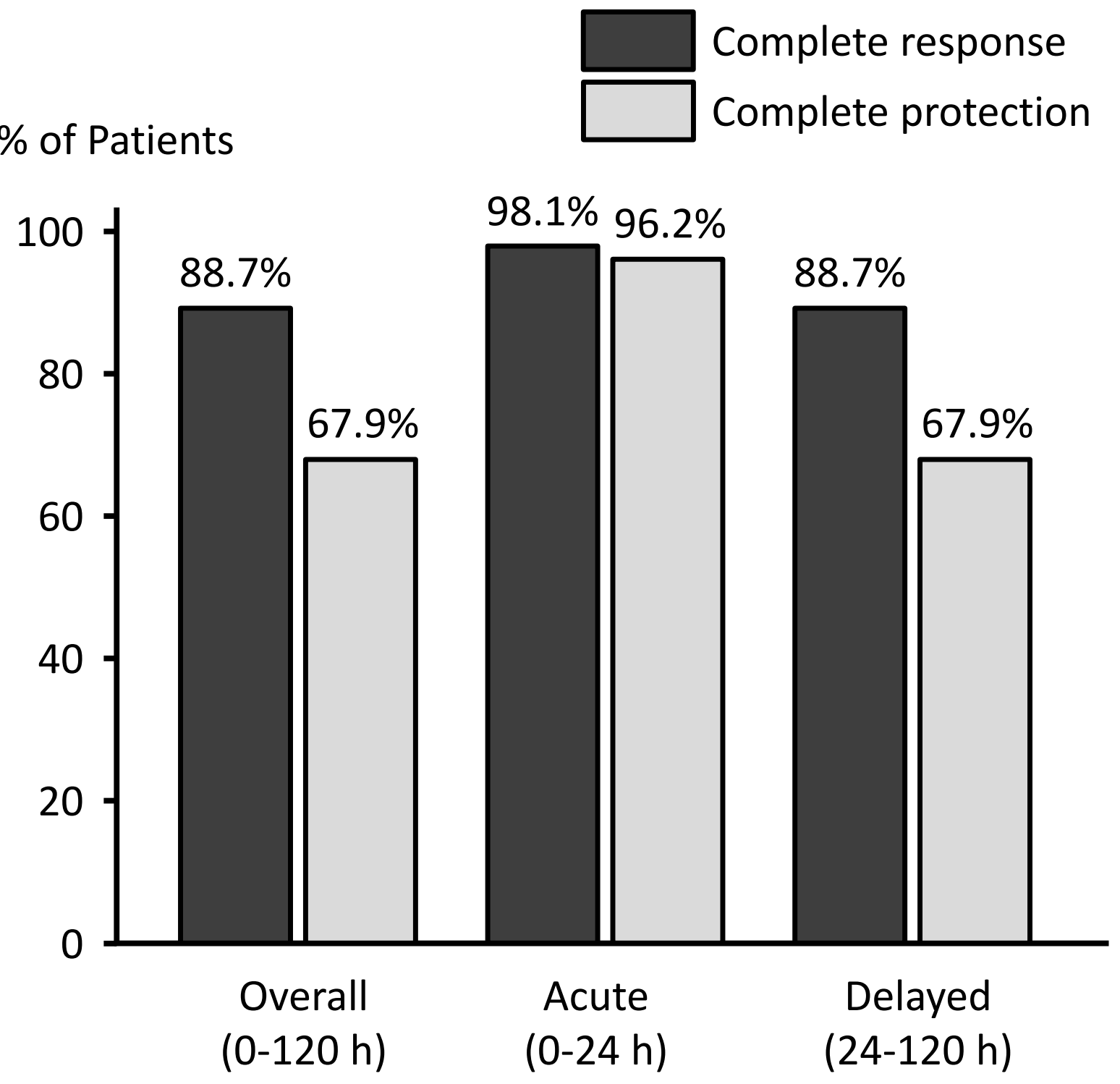
Figure 2. Decrease in diet intake compared to that before initiation of chemotherapy

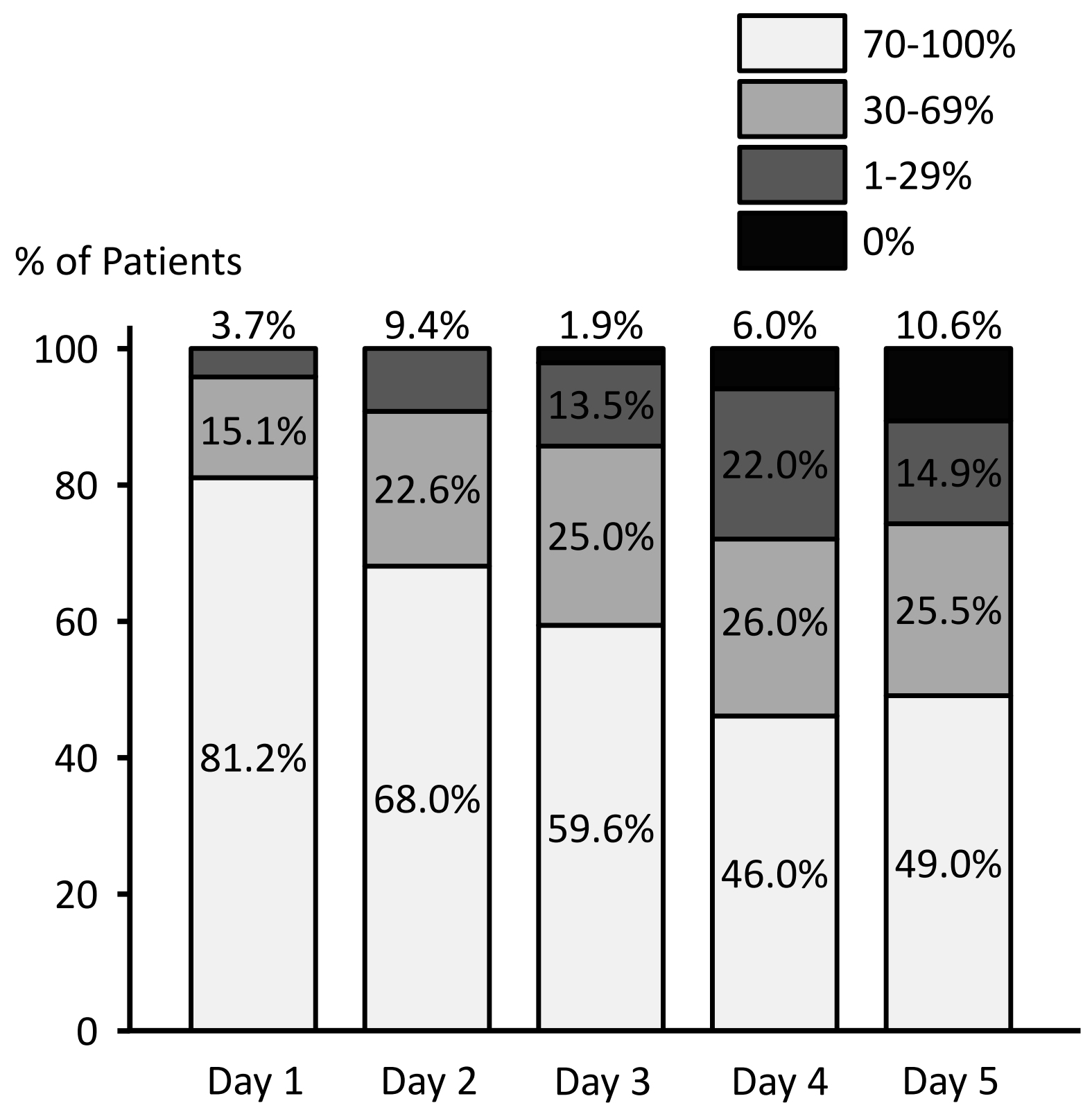

